\title{
LEETT
}

ISSN: 2277-3754

ISO 9001:2008 Certified

International Journal of Engineering and Innovative Technology (IJEIT)

Volume 10, Issue 9, March 2021

\section{Adaptive Colour Space based Robust Image Watermarking Using Serial DWT-HD-SVD domain}

\author{
Sandeep Singh Rathord, Manish Rai \\ RKDF Bhopal, India
}

\begin{abstract}
Watermarking is used for the authentication and protection of digital image data. The color image watermarking is a challenging task and may endup to be lengthy. Various transformations based methods are used forenhacingwatermarkrobustnessand to keep itinvisible. Robustness of watermarking methods have been improved using the hybrid DWT-SVD domain. In this paper an improved hybrid transformation method is prpoposed, using the combination of the Hesenberg Decomposition (HD) with the existing DWT-SVD method. The LAB colour space is adopted at the pre-processing stage for the entropy maximization. The entropy analysis is used for justification of the colour space adaptation.Performance of the existing and proposed method is tested on colour images using parametric analysis of normalized correlation (NC),Peak signal to noise ration (PSNR) and SSIM.Efficiency of existing watermarking methods may detoriate undervarious attacks. The Impact of the various watermark attacks are also evaluated over the extraction quality.
\end{abstract}

Keywords-Image Watermarking, Invisibility, DWT, SVD, Hesenberg Decoposition, PSNR, SSIM. Watermark attacks.

\section{INTRODUCTION}

The wide range of the digital true color images are stored over theInternet introduces the need image data protection. DWT-SVD based watermarking methods are widely being used in literature [1] for improving the roboustness. An watermarking method must be roboustagaist the various attacks. The eprime concern of this paper is to focus on color images security. Watermarking method is used for securingtrue color imagery data from copy rights and protecting them from various attacks viz. Noise, median filtering, cropping, and rotation attacks.In order to improve the robustness of the watermarking, an improved hybrid transformation method is prpoposed using the combination of the Hesenberg Decomposition (HD) with the existing DWT-SVD method [2]. Various scaning modalities enable to capture colourtrue color images [2].The color fetures are more precesiely understood and segmented by the hunman eyes.For improving the performance an adaptive LAB colour space adoption is proposed for more robostness. As it is expected that entropy is improved in LAB space.

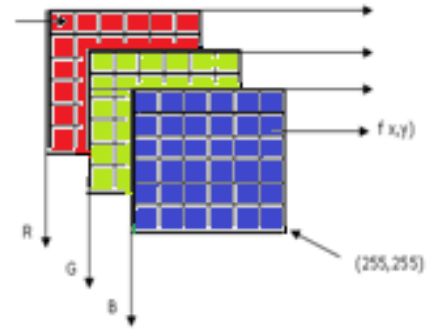

Fig.1. Color RGB image representation [3]

Manuscript received: 26 February 2021

Manuscript received in revised form: 25 March 2021

Manuscript accepted: 10 April 2021

Manuscript Available online: 15 April 2021
A color image is a 3D array of the size $m \times n \times 3$, the coordinates $\mathrm{x}$ and $\mathrm{y}$ can have values between $(0,0)$ to $(255,255)$ corresponding to RGB color space.

Therefore, these coloured images are more frootfuland valuable than similar gray level images. The color image is represented in matrix form as in Figure 1.The pixel value of a color image $f$ maylies between $0-255$ for all RGB domains and brightness is given by;

$$
f(x, y)=0 \leq g \leq 2^{n-1}
$$

where $\mathrm{n}$ is the number of bits where 0 is black and 255 means pure white.The watermking needs to stisfy the property of invisibility to have good visual quality. Usually invisibility is more in the transform domain methods thus they are our prime concern in this paper. Transform domain based methods are further classified as in Figure 2.

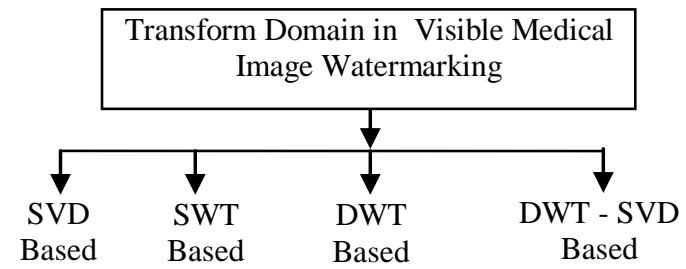

Fig.2. Classification chart of transform domaintrue color image watermarking methods

Transformation based true color image watermarking methods as entioned in Figure 2 are widely used to to improve robustness and invisibility of the watermark. Discrete Wavelet Transfor (DWT) and Singuler Value Decomposition (SVD) are widely used with all three mentioned methodologies for adding robustness.

This paper reviews the limitations of existingtransformation based true color image watermarking methodsdesigned forprotection oftrue color imaging data in section 2. The wa2velet based watermarking methods and basic concepts of DWTdecomposition and its benefits are briefly explored in section 3.In next section concept of color spaces andSVDalong with there properties are discused.In this paper the Lab color space is proposed to opt for watermaringand expected to improve entropy.

The luminance componentLof adopted LAB color space is separated and chroma components(A and B) are preserved for reproduction. Implementing the ColorImage Watermarking (CIW)in LAB color space shows the improvement over previous method [2], the proposed methodology is explained in section 5. Finally the results of CIW and entropy analysis along withwatermark embedding and reconstructin are presented. the entropy and PSNR as quality measures, and conclusions are discussed in the section 6 . 


\section{DVETT}

ISSN: 2277-3754

ISO 9001:2008 Certified

International Journal of Engineering and Innovative Technology (IJEIT)

Volume 10, Issue 9, March 2021

\section{RELATED WORK}

Numerous watermarking methods were designed in the literature. This paper focuses to review the transform based methodologies based on hybrid SVD and DWT domain, Ying Huang et al [1] have proposed an spread spectrum nased method for dopting the empeding parameters. They tried to improve the gurentee of PSNR perfroamcne. Junxiu Liu et al [2] have proposed an new hybrid approach of combining the HD with DWT-SVD doain for embedding the watermark. they also evaluated thrfroamcneunsder various attacks by varying the scaling parameter for embedding. They also analysed the performance under different watermark sizes. But there method shows the gray level results.Y. Tan, et al [3] have used channel coding method for embedding thusee SVD based watermarking for copyright protection application. watermark in the $\mathrm{Y}-\mathrm{Cb}-\mathrm{Cr}$ colot space. They have embedded the watermark using the SVD of the HL wavelet sub-band. Further DWT-SVD based methods are explained in $[4,5$, and 6] respectively.Ramanand Singh. Paresh Rawat have proposed to use the Stationary Wavelet transform (SWT) for embedding the watermark using Edge detection. R. Rykaczewski et al [8] have used used the SVD for copy right protection.Q. Su et al [13 and 14] have used the HD decomposition for designing the watermarking method for images. Thus this papertakes advantage of Q. Su approach forimprovingroboustness.

\section{WAVELET DECOMOPOSITIONS}

Discrete wavelet transform (DWT) is widely being used fro improving the robustness of the watermarking methods. DWT down samples the image using low pass and high pass filter banks to multi resolution subbands as LL, LH,HL, and HH. An example of the DWT decomposition of the input image uptolevel 1 and level 2 are givein in the Figure 3.It is clear that thetransform domain DWT offers more robustness due to better embedding options.
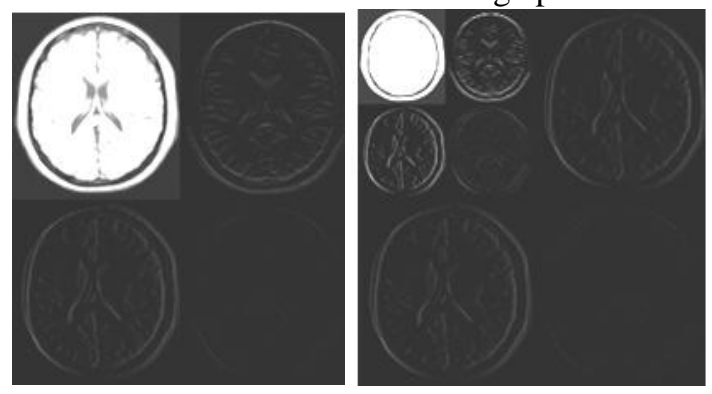

Fig.3. DWT decomposition example images a) First level, b) Second Level 2

\section{HD AND SVD FOR IMAGE PROCESSING}

HD:It is representing the Hessenberg Decomposition, is a matrix decompositions usedfor only square matrix decomposition $[13,14]$. A $n \times n$ square size matrix $X$ may be decomposed using HD as given in the Eq.

$$
P H P^{T}=H D(X) ;
$$

SVD: The concept of singular value decomposition (SVD) is widely used for watermarking the images.Using the SVDcan decomposed an image matrixI of size $x * y$ as product of three matrices given by

$\mathrm{I}=U * S * V^{T}$

Finalythe Smatrix is singular values.

\section{PROPOSED WATERMARK EMBEDING}

The proposed watermarking method embeds the watermark lpgo in decomposed DWToefficients followed by the HD decomposition and then replacing SVD values. There are two major contributions of this methods are: It adopts the color component adaptively based on entropy analysis. While as an improvement over [2], paper makes method more fast the DWT level are kept fixed to 2 by adjusting the size of the watermark

\section{Watermark Embeding}

The Flow Chart of proposed watermark embedding method is presented in Figure 5. The dual key are used to embede the watermark serialy every time different text numeric key is used for embedding. Sequential procedure for embedding the watermark is presented as follows;

1. Read the input true color images or cover image.

2. Convert RGB to LAB color components.

3. Use the L level DWT to tune the watermark ad cover image size.

4. Calculate HD decomposition.

5. Find the SVD values on $H$ component.

6. Generate watermrked image by replacing SVD of coverimage from SVD of the Logo.

7. Take the inverse SVD, HD and DWT to reconstruct the watermarked true color image then merge colors.

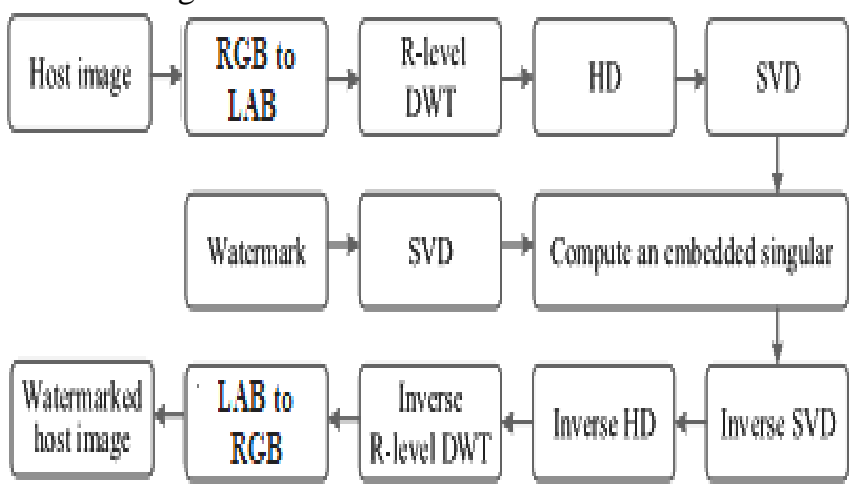

Fig.4. Block diagram of proposed watermarking methodology

\section{RESULTS AND DISCUSSIONS}

In this section the expected outcomes of the proposed watermarking methodsare presented. Also in sexond part odsextion the parametric evaluation of the performance is presented. The various inputcolouriimages used for the current studuy are presented in the Figure 5. The first stage basic watermarking and extraction results are shown in the Figure 6 for Lena image with L component. The efficiency of the waremarkretrival can be clerly observed for the Figure. 


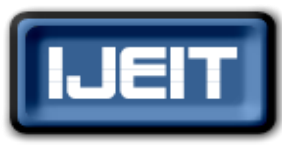

ISSN: 2277-3754

ISO 9001:2008 Certified

International Journal of Engineering and Innovative Technology (IJEIT)

Volume 10, Issue 9, March 2021
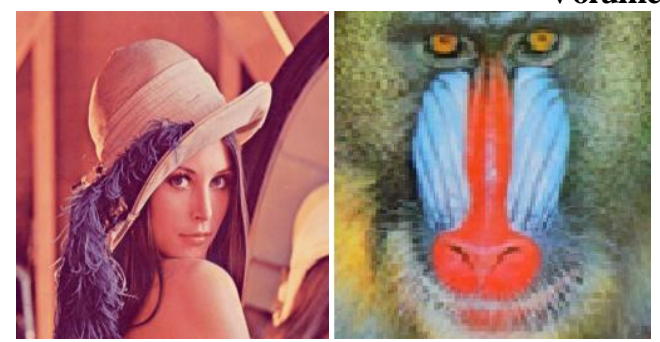

a) Lena iamge

b) Baboon Image
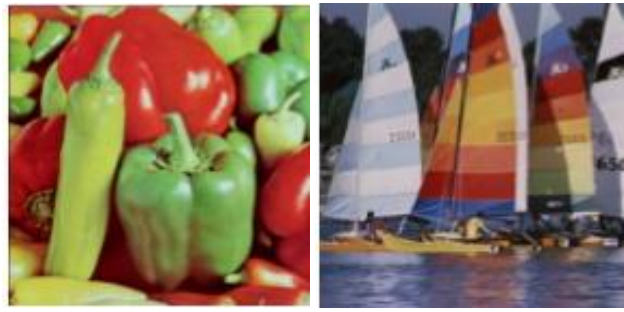

c) peppers image d) Yatch image

Fig.5. Input images used for study.

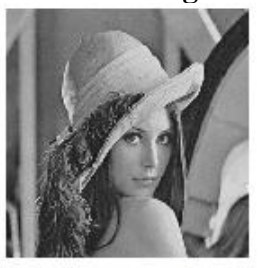

a) Cover image

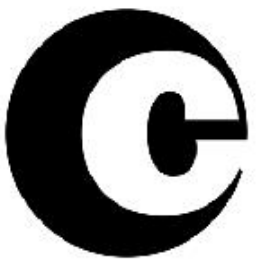

c) Watermark logo

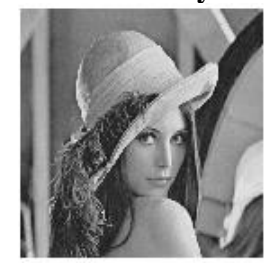

b) Watermarked image DWT-HD-SVD method $\alpha=$ Sharpening attack

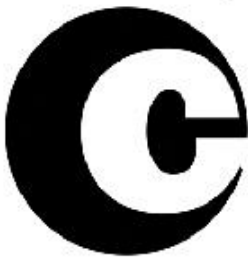

d) Extracted watermark
Fig.6. Results of watermarking without any attack and extracted images.

In order for performancecomperision watermarking results of the Junxiu Liu at al [2] and the our proposed method with different logo size are compared in Figure 7

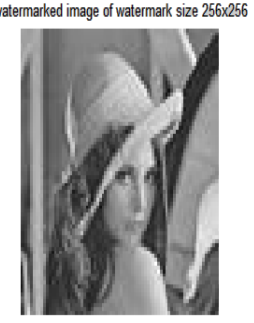

PSNR= 38.1621 SSIM $=0.9992$

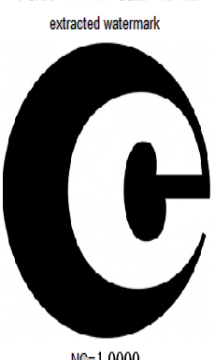

$\mathrm{NC}=1.0000$
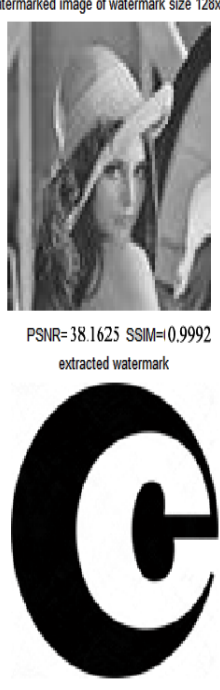

NC $=1.0000$
PSNR $=38.1625$ SSIM $=10.9992$

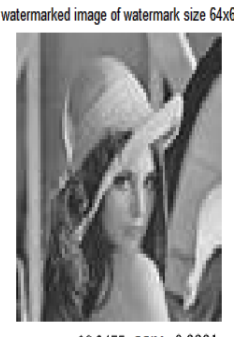

PSNR $=38.2477$ SSIM $=0.9991$

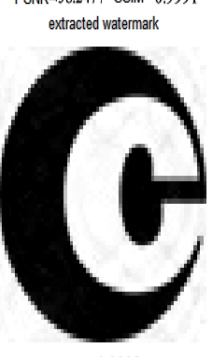

$\mathrm{NC}=1.0000$ a) watermarking results of the Junxiu Liu at al [2]
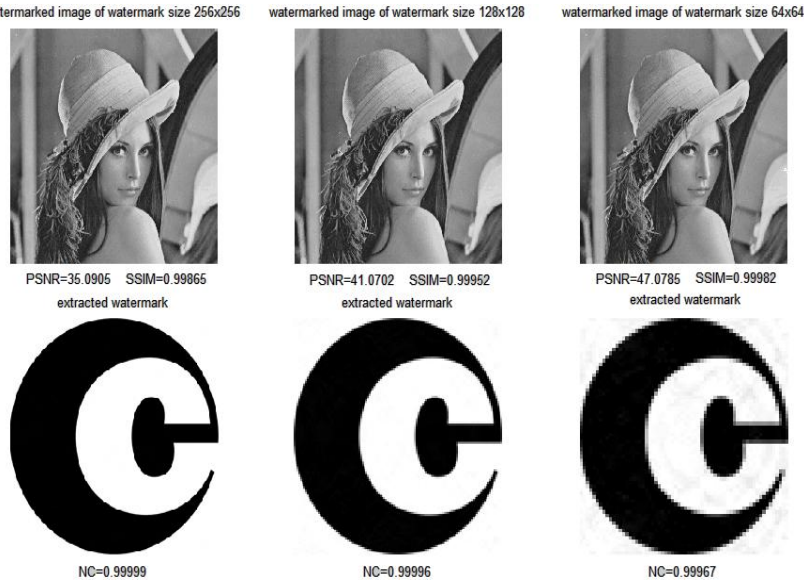

b) Watermarking with proposed Lab space

Fig.7. Results comparison of watermarked and extrectedlogo images with proposed method in LAB space

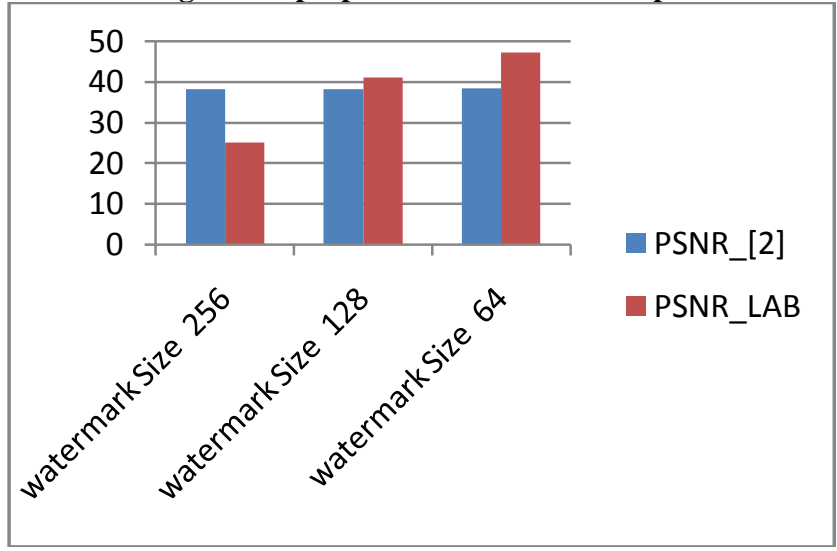

Fig.8.Comparision of the PSNR perfroamcne.

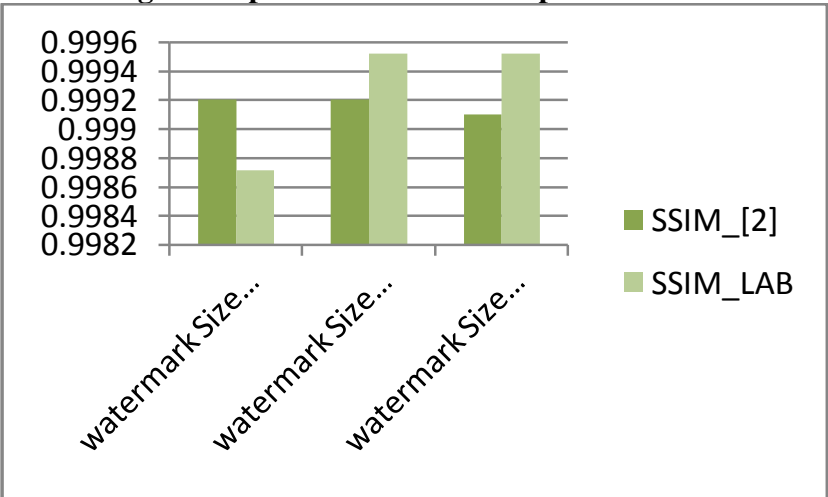

Fig.9.Comparision of the SSIM perfroamcne

The parametric perfromace of the comparison shown in thgure 7 are evaluated and ploted in Bar chart form for the peak signal to noise ration 9 PSNR) and the structure similarity index measure (SSIM) in the Figure 8 and Figure 9 respectivly.

It is observed that the propsed method out perfromas then existing one for different watermark size. Of $128 \times 128$ and 64 x64. Ther is asignificant improvement in the SSIM performance.

\section{Perfromance Under Attcaks}

In order to show the performance under the various attacks the this section the PSNR performance is varied under the different scaling parameter fembedding and plotedagaist different attacks. 


\section{WEIT}

ISSN: 2277-3754

ISO 9001:2008 Certified

\section{International Journal of Engineering and Innovative Technology (IJEIT)}

Volume 10, Issue 9, March 2021

Resuts of the PSNR comparision for different attacks with proposed method are presented in the Figure 10. In this paper Gaussian filter, Median filters, Gaussian noise, Average Filter and the Motion Blur attacks are considered for the evaluating the.performance under the presence of Gaussian filter attack for different attacks are presented in the Figure 11.

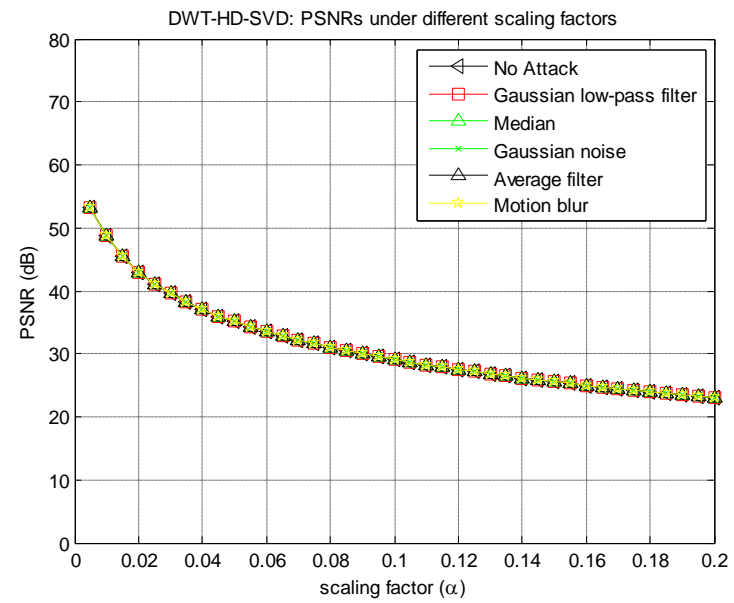

Fig.10. Results of the PSNR comparision for different attacks with proposed method.
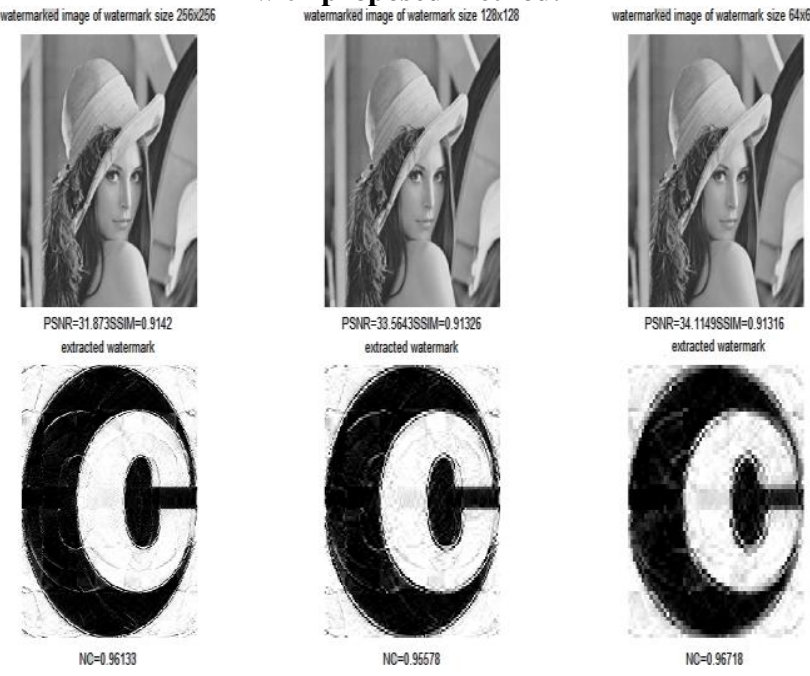

Fig.11. Performance under Gaussian filter attack.

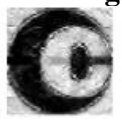

0.8322

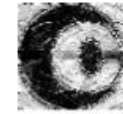

0.8569

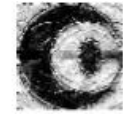

0.9147

With unxiu Liu ]2]
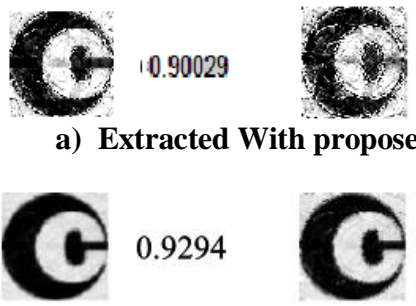

$=0.84456$

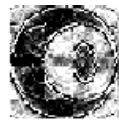

0.78997

a) Extracted With proposed method for Motion blur

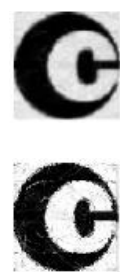

0.9294

0.9539

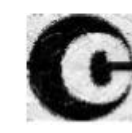

0.9911

0.95524

With unxiu Liu ]2]

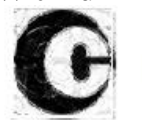

0.9462

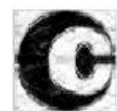

0.95444

b) Extracted With proposed method for Average Filter

Fig.12. Comparision of the performance under the different attacks for proposed and existing method.
The performance improvement with the proposed LAB color space based watermarking can be clearly observed from the comparison of perfroamcne under the different attacks. For proposed and existing method. As shown in Figure 12.

\section{CONCLUSION AND FUTUREWORK}

In this paper an modified watermarking method is proposed for improving the roboustness. As an modification the watermarking is implemented in the LAB color space instead of RGB space.

The watermarking is implemented using the DWT-HDSVD domain. The scaling parameter alpha is varied for performance evaluatin. The performance of the proposed method is compared witht the existing method under the presence of different attacks.

It is observed that the propsed method out performs as then existing one for different watermark size. of $128 \times 128$ and 64 x64. There is a significant improvement in the SSIM performance.it is observed that for the motion blur attack the perfromace of proposed method for 256x256 size is improved for $\mathrm{NC}$ from 0.8322 to 0.9003 and for the average filter 0.9294 to 0.9552 . In future various embedding rules and the wavelet filters can be evaluated for performance.

\section{REFERENCES}

[1] Y. Huang, B. Niu, H. Guan and S. Zhang, "Enhancing Image Watermarking With Adaptive Embedding Parameter and PSNR Guarantee," IEEE Transactions on Multimedia, vol. 21, no. 10, pp. 2447-2460, Oct. 2019.

[2] Junxiu Liu, Jiadong Huang, Yuling Luo, Lvchen Cao,Su Yang, Duqu Wei, and Ronglong Zhou, "An Optimized Image Watermarking Method Based on HD and SVD in DWT Domain”, IEEE Access, vol. 7, pp. 80849-80860, July 2019.

[3] Y. Tan, J. Qin, X. Xiang, W. Ma, W. Pan and N. N. Xiong,"A Robust Watermarking Scheme in YCbCr Color Space Based on Channel Coding,"IEEE Access, vol. 7, pp. 25026-25036, 2019.

[4] K. Chaitanya, E. Srinivasa Reddy, Dr K. Gangadhara Rao,"Digital Color Image Watermarking In RGB Planes Using DWT-DCT-SVD Coefficients",International Journal of Computer Science and Information Technologies, Vol. 5 (2),pp-2413-2417, 2014.

[5] Ling-Yuan Hsua and Hwai-Tsu Hub "Blind watermarking for color images using EMMQ based on QDFT", Expert Systems with Applications, Volume 149, 1, pp:1-16, July 2020.

[6] Chih-Chin Lai, and Cheng-Chih Tsai, "Digital Image Watermarking Using Discrete Wavelet Transform and Singular Value Decomposition",IEEE Transactions On Instrumentation and Measurement, Vol. 59, No. 11, pp:30603063,Nov, 2010.

[7] Ramanand Singh. Paresh Rawat, Piyush Shukla,"Robust True color Image Authentication using 2-D Stationary Wavelet Transform and Edge Detection",IET International Conference ICBISP China, pp:54-59,2017.

[8] R. Rykaczewski,“An SVD-Based Watermarking Scheme for Protecting Rightful Ownership", IEEE Transactions on Multimedia, vol. 9, no. 2, pp. 421-423, Feb. 2007. 
ISSN: 2277-3754

ISO 9001:2008 Certified

International Journal of Engineering and Innovative Technology (IJEIT)

Volume 10, Issue 9, March 2021

[9] Apeksha Tiwari, Virendra Singh "Digital Image Watermarking Using DWT and Shift Invariant Edge Detection", International Journal of Computer Technology and Electronics Engineering (IJCTEE) Volume 3, Issue 6, pp:66-71, December 2013.

[10] Poonam Kadian, Nidhi Arora,Shaifali M. Arora,"Performance Evaluation of Robust Watermarking Using DWT-SVD and RDWT-SVD",6th International Conference on Signal Processing and Integrated Networks (SPIN), 987-991, 2019.

[11] Yuqi He ,Yan Hu,“A Proposed Digital Image Watermarking Based on DWT-DCT-SVD", 2nd IEEE Advanced Information Management,Communicates,Electronic and Automation Control Conference (IMCEC), pp: 1214-1218, 2018.

[12] I. A. Ansari, C. W. Ahn and M. Pant,"On the security of Block-based SVD image watermarking in spatial and transform domains", 2018 International Conference on Digital Arts, Media and Technology (ICDAMT), Phayao, Thailand, pp. 44-48, 2018.

[13] Q.Su and B. Chen,"A novel blind color image watermarking using upper Hessenberg matrix," AEU-Int. J. Electron. Commun., vol. 76, no. 6, pp. 64-71, 2017.

[14] Q. Su,"Novel blind colour image watermarking technique using Hessenberg decomposition”, IET Image Process., vol. 10, no. 11, pp. 817-829, Nov. 2016.

[15] SuneshVinita Malik, Neeti Sangwan, Sukhdip Sangwan,"Digital Watermarking using DWT-SVD Algorithm", Advances in Computational Sciences and Technology, Volume 10, Number 7 pp. 2161-2171, 2017.

[16] K. Vidya, T Sujitrha, "Robust Dwt-Svd Based Blind Watermarking For Dibr 3d Video",2nd International Conference on Computer, Communication, and Signal Processing (ICCCSP), pp: 1-6, 2018. 